\title{
Facile fabrication and characterization of chitosan-based zinc oxide nanoparticles and evaluation of their antimicrobial and antibiofilm activity
}

\author{
Gurpreet Singh Dhillon • Surinder Kaur • \\ Satinder Kaur Brar
}

Received: 22 December 2013/ Accepted: 15 May 2014/Published online: 6 June 2014

(C) The Author(s) 2014. This article is published with open access at Springerlink.com

\begin{abstract}
The present investigation deals with the facile synthesis and characterization of chitosan (CTS)-based zinc oxide $(\mathrm{ZnO})$ nanoparticles (NPs) and their antimicrobial activities against pathogenic microorganisms. ZnO-CTS NPs were synthesized through two different methods: nano spray drying and precipitation, using various organic compounds (citric acid, glycerol, starch and whey powder) as stabilizers. Both the synthesis methods were simple and were devoid of any chemical usage. The detailed characterization of the NPs was carried out using UV-Vis spectroscopy, dynamic light scattering particle size analysis, zeta potential measurements and scanning electron microscopy, which confirmed the fabrication of NPs with different shapes and sizes. Antimicrobial assay of synthesized ZnO-CTS NPs was carried out against different pathogenic microbial strains (Candida albicans, Micrococcus luteus and Staphylococcus aureus). The significant $(p<0.05)$ inhibition of growth was observed for both $M$. luteus and $S$. aureus with ZnO-CTS NPs (with a concentration ranging from 0.625 to $0.156 \mathrm{mg} / \mathrm{ml}$ ) as
\end{abstract}

G. S. Dhillon · S. K. Brar $(\bowtie)$

Centre-Eau Terre Environnement/Centre for Water, Earth and

Environment, Institut national de la recherche scientifique,

Université du Québec, 490, Rue de la Couronne, Québec,

QC G1K 9A9, Canada

e-mail: satinder.brar@ete.inrs.ca

G. S. Dhillon

Department Agricultural, Food and Nutritional Sciences (AFNS), University of Alberta, Edmonton, AB T6G 2P5, Canada

S. Kaur

Department of Mycology and Plant Pathology, Institute of Agricultural Sciences, Banaras Hindu University (BHU),

Varanasi 221005, India compared to control treatment. $\mathrm{ZnO}-\mathrm{CTS}$ NPs also showed significant biofilm inhibition activity $(p<0.05)$ against $M$. luteus and $S$. aureus. The study demonstrated the potential of $\mathrm{ZnO}-\mathrm{CTS}$ NPs as antimicrobial and antibiofilm agents.

Keywords Antimicrobial - Antibiofilm - Chitosan · Nanoparticles · Nano spray dryer, precipitation method, zinc oxide
Abbreviations
CA Citric acid
CTS Chitosan
NPs Nanoparticles
SEM Scanning electron microscope
PDI Polydispersity index

\section{Background}

Metal oxide nanoparticles (NPs), such as zinc oxide $(\mathrm{ZnO})$, have been found to exhibit interesting properties, such as large surface-to-volume ratio, high surface reaction activity, high catalytic efficiency, and strong adsorption ability [1-3], that make them potential candidates for various applications. $\mathrm{ZnO}$ is an inorganic material with several advantages, such as wide band gap $(3.34 \mathrm{eV})$, semiconductor with a high exciton binding energy $(60 \mathrm{meV})$, high isoelectric point (9.5), and fast electron transfer kinetics $[4,5]$. All these features suggest the feasibility for synthesis of special $\mathrm{ZnO}$ nanostructures. Nano $\mathrm{ZnO}$ can be synthesized in many forms: rods, wires, whiskers, belts, bipods, tetrapods, tubes, flowers, propellers, bridges, and cages [6-8]. Nanostructured $\mathrm{ZnO}$ 
has great potential for many practical applications, such as dye sensitized solar cells, piezo electric transducers, UV-light emitters, chemical and gas sensors and transparent conductive coating [9]. It also exhibits intense ultraviolet absorption and can potentially be utilized as UV-shielding materials and antibacterial agents [10]. $\mathrm{ZnO}-\mathrm{NPs}$ have been prepared by techniques, such as the sol-gel method [11, 12], precipitation [13], hydrothermal synthesis [14], and spray pyrolysis [15].

Recently, chitosan (CTS) hybrid materials including conducting polymers, metal NPs and oxide agents have been developed with excellent properties of individual components and outstanding simultaneous synergistic effects [16]. Currently, the research on the combination of CTS and metal oxide has focused on titanium dioxide $\left(\mathrm{TiO}_{2}\right)$, as it possesses excellent photo catalytic performance and is stable in acidic and alkaline solvents. In parallel with $\mathrm{TiO}_{2}, \mathrm{ZnO}$ also has similar band gap and antibacterial activity [16]. The cationic nature of CTS makes it possible to adhere to the negatively charged surface, such as bacterial cell membranes. CTS is soluble in dilute acidic solutions and interacts with polyanions to form complexes and gels. CTS is innocuous and possesses antibacterial and antifungal properties [17, 18]. CTS has tremendous ability to form metal complexes with $\mathrm{Zn}$ metal [19]. Owing to the presence of amine and hydroxyl groups on CTS, currently ZnO-CTS complex attracted great interest for its potential use as UV protector and medicament.

However, due to the extremely high reactivity, the initially formed $\mathrm{ZnO}$ NPs tend to react rapidly with the surrounding media or agglomerate, resulting in the formation of much larger ( $\mu \mathrm{m}$ to $\mathrm{mm}$ scale) particles or flocs. In the process, they rapidly lose their bioactivity. In order to prepare more stable and active ZnO NPs, studies have been carried out either by the fabrication on the surface of NPs by organo-alkoxysilane [20] or by grafted polymers [21]. However, only few studies have been carried out on the surface modification of NPs to create a stable dispersion in aqueous medium. Various organic compounds, such as citric acid (CA), glycerol, starch and whey powder (contains lactose moiety), among others can be used as capping agents. These water-soluble organic compounds serve as a stabilizer and dispersant that prevents the resultant NPs from agglomeration, thereby prolonging their reactivity and maintaining the physical integrity. Moreover, these organic compounds are safe and innocuous.

The present work was carried out with following objectives: (1) facile synthesis and characterization of ZnO-CTS NPs with different stabilizers using nano spray drying and precipitation method. Various organic compounds, such as CA, glycerol, starch and whey powder, were used as capping agents to stabilize the NPs formulation and; (2) application of ZnO-CTS NPs as antimicrobial and antibiofilm forming agents against pathogenic microbial strains, e.g., Candida albicans, Micrococcus luteus and Staphylococcus aureus. These strains were selected due to their widespread occurrence and resulting health hazards. $C$. albicans is a causal agent of opportunistic oral and genital infections in humans [22]. Systemic fungal infections (fungemias) by C. albicans have emerged as major causes of morbidity and mortality in immuno-compromised patients. Candida albicans forms biofilms on the surface of implantable medical devices, as a consequence hospitalacquired infections by $C$. albicans have become a cause of major health concerns. Micrococcus luteus is a Grampositive, spherical, saprotrophic bacterium that belongs to the family Micrococcaceae. Micrococcus luteus is found in soil, dust, water and air, and as part of the normal flora of the mammalian skin. It also colonizes the human mouth, mucosae, oropharynx and upper respiratory tract. Micrococcus luteus shows higher potential for biofilm formation. Staphylococcus aureus is a facultative anaerobic Gram-positive coccal pathogenic bacterium belonging to Staphylococcaceae family. Staphylococcus aureus is the prevalent cause of food intoxication and can cause a wide range of illnesses, from minor skin infections to life-threatening diseases, such as pneumonia, meningitis, osteomyelitis, endocarditis, bacteremia and sepsis. It is one of the five most common causes of nosocomial infections and is often the cause of postsurgical wound infections [23].

\section{Materials and methods}

\section{Chemicals}

Yeast Peptone Glucose (YPG) medium, tryptic soya broth and agar were purchased from Fischer scientific. Zinc oxide $(\mathrm{ZnO})$ and $\mathrm{CA}$ were supplied by Sigma-Aldrich (Ontario, Canada). CTS (molecular weight- 600-800 kDa and degree of deacetylation (DD) $>90 \%$ and viscosity 200-500 mPa s) and starch (soluble, ACS grade) were purchased from Fisher Scientific (Ontario, Canada). Whey powder (whey permeate) was purchased from Agropur Cooperative, Québec, Canada. Milli-Q water was prepared in the laboratory using a Milli-Q/Milli-RO Milli pore system (Milford, MA, USA). Glycerol was purchased from Labmat Inc. Québec, Canada. The composition or properties of whey powder were as follows: carbohydrates (mainly as lactose) $>82.0 \%$; protein $\leq 3.5 \%$; fat $<0.3 \%$; ash content 8.0-9.0\%; moisture $<5.0 \%$; pH (10\% solution) 6.1 and titrable acidity of $0.12 \%$. 
Preparation of ZnO-CTS NPs

\section{Nano spray drying method}

The synthesis of ZnO-CTS NPs was carried out using nano spray dryer (B-90 BUCHI, Switzerland). NPs were prepared by dissolving $0.75 \mathrm{~g}$ of $\mathrm{ZnO}$ powder in $100 \mathrm{ml}$ of $1 \%(\mathrm{v} / \mathrm{v})$ acetic acid. This led to the dissociation of $\mathrm{ZnO}$ to zinc cations. $1 \mathrm{~g}$ of CTS was added to this solution. After fully dissolving the CTS, different organic compounds [1\% (w/v) CA; $0.5 \%(\mathrm{w} / \mathrm{v})$ whey powder; $0.5 \%(\mathrm{w} / \mathrm{v})$ soluble starch and $0.5 \%$ (w/v) glycerol] were added as stabilizers in various treatments. For comparison, control of ZnO NPs and ZnO-CTS NPs was prepared in the absence of stabilizer following the similar procedure. The process conditions were as follows: spray speed was $100 \%$; inlet and head temperature was set at $120 \pm 1{ }^{\circ} \mathrm{C}$. The pump speed was adjusted on standard mode of 1 and gas pressure was adjusted at $40-45 \mathrm{hPa}$ which corresponds to a gas flow of around $150-160 \mathrm{l} / \mathrm{min}$. The NPs formed were manually collected using particle collection paper.

\section{Precipitation method}

The similar procedure as nano spray dryer was followed and after making the solution containing $\mathrm{ZnO}-\mathrm{CTS}$ in the absence or presence of stabilizer, the mixture was sonicated for $30 \mathrm{~min}$. With magnetic stirring, $1 \mathrm{M} \mathrm{NaOH}$ was added until the solution attained $\mathrm{pH} 10$. The samples were heated in water bath at $80 \pm 1{ }^{\circ} \mathrm{C}$ for about $3 \mathrm{~h}$ followed by frequent washing with distilled water and lyophilization using a freeze dryer (ScanVac Cool Safe, LaboGene, Lynge, Denmark).

\section{Analytical measurement}

Viscosity of different samples was measured using a rotational viscometer Brookefield DVII PRO + (Brookfield Engineering Laboratories, Inc., Stoughton, MA, USA) equipped with Rheocalc32 software. The viscosity data acquisition and analysis were carried out using Rheocalc V2.6 software (B.E.A.V.I.S.—Brookfield Engineering Advanced Viscometer Instruction Set). All measurements were performed at $25 \pm 1{ }^{\circ} \mathrm{C}, 36.69 \mathrm{~s}^{-1}$ shear rate and viscosity was referred to as "apparent viscosity". $\mathrm{pH}$ of the different metal oxide solutions was measured with the $\mathrm{pH}$ meter equipped with glass electrode.

\section{Characterization of NPs}

\section{$U V-V i s$ spectrum}

Absorption spectra of the NPs in aqueous suspension were recorded using a UV-Vis spectrophotometer (UV 0811
M136, Varian, Australia) at 200-600 nm. The optical path length of the cuvettes used in all the experiments was $1 \mathrm{~cm}$. The absorption spectra were recorded at room temperature.

Size, zeta potential measurements and scanning electron microscope (SEM)

The mean size, size distribution and zeta potential measurements of the ZnO-CTS NPs were performed by Nano Zetasizer (Nano series, Malvern instruments Ltd., Worcestershire, UK) equipped with multipurpose titrator (MPT-2). The morphology of the synthesized ZnO NPs was examined by a scanning electron microscope (Model: Carl Zeiss EVO ${ }^{\circledR} 50$ smart SEM) with a magnification of $10.0 \mathrm{~K} \mathrm{X}$, working distance (WD) $20 \mathrm{~mm}$, secondary electrode 1 (SE 1) detector and EHT $10 \mathrm{kV}$. Samples were coated with gold before SEM testing.

Assessment of in vitro antimicrobial and antibiofilm activity of $\mathrm{ZnO}-\mathrm{CTS}$ NPs

In this study the fungal strain, Candida albicans CCRI 10,345, and bacterial strains, Micrococcus luteus CCRI 16,025 and Staphylococcus aureus CCRI 20,630, were used to assess antimicrobial and antibiofilm activity of ZnO-CTS NPs formulations. The microbial strains were cultured for $48 \mathrm{~h}$ using Yeast Peptone Glucose (YPG) medium agar plates (C. albicans) and tryptic soya agar plates (M. Luteus and S. aureus).

The qualitative antimicrobial assay

The in vitro qualitative screening of the different $\mathrm{ZnO}-$ CTS NPs was performed using agar diffusion method. The filter paper discs (5 $\mathrm{mm}$ diameter) were dipped in each filter sterilized tested sample (NPs concentration of $5 \mathrm{mg}$ / $\mathrm{ml}$ ) and were placed in Petri dishes with yeast extract, peptone and glucose (YPG) (for fungi) and tryptic soya agar (for bacteria) medium previously seeded with the bacterial inocula. The inoculated plates were incubated for $24 \mathrm{~h}$ at $30 \pm 1{ }^{\circ} \mathrm{C}$. Antimicrobial activity was assessed by measuring the inhibition zone diameter $(\mathrm{mm})$. Based on the qualitative assay results, only the bacterial strains susceptible to tested samples have been further tested in the quantitative assay using liquid medium.

The quantitative antimicrobial assay of the minimal inhibitory concentration

The quantitative assay was performed in liquid medium with twofold serial dilutions of NPs solution (ranging between 5.0 and $0.156 \mathrm{mg} / \mathrm{ml}$ ) using susceptible strains assessed through qualitative assays. The assay was 
performed in 96-well plates using tryptic soy broth medium. In brief, $200 \mu \mathrm{l}$ volume of nutrient medium was poured in each well and inoculated with $20 \mu \mathrm{l}$ inocula of $48 \mathrm{~h}$ grown cultures.

The microplates were incubated at $30 \pm 1{ }^{\circ} \mathrm{C}$ for $24 \mathrm{~h}$. The antimicrobial activity was quantified by measuring the optical density at $600 \mathrm{~nm}$ and minimum inhibitory concentration (MICs) was read as the last concentration of the tested compounds, which inhibited the microbial growth.

\section{Antibiofilm activity}

The antibiofilm activity was performed using the micro titer method. To do so, the microbial strains were grown in the presence of twofold serial dilution of NPs (ranging between 5.0 and $0.156 \mathrm{mg} / \mathrm{ml}$ ) in liquid nutrient medium distributed in 96-well plates. In brief, $200 \mu$ medium with known concentrations of tested sample was inoculated with $20 \mu \mathrm{l}$ bacterial suspension and incubated for $24 \mathrm{~h}$ at $30 \pm 1{ }^{\circ} \mathrm{C}$ for bacterial strains. At the end of the incubation time, the plastic wells were emptied, washed three times with phosphate buffer saline (PBS), fixed with cold methanol, and stained with $1 \%$ violet crystal solution for $30 \mathrm{~min}$. The biofilm formed on plastic wells was resuspended in $30 \%$ acetic acid. The intensity of the colored suspension was assessed by measuring the absorbance at $492 \mathrm{~nm}$ [24]. The last concentration of the tested compound that inhibited the development of microbial biofilm on the plastic wells was considered as the MICs of the biofilm development and was also expressed in $\mathrm{mg} / \mathrm{ml}$ [25].

\section{Statistical analysis}

The quantitative antimicrobial assay and biofilm inhibition assay were performed in triplicates and values are given as average \pm SD. The data were statistically analyzed using the multiple analysis of variance (ANOVA) by SPSS (16.0 version). To determine the significance of difference between different treatments and concentrations of $\mathrm{ZnO}$ CTS NPs for antimicrobial and biofilm inhibition of $M$. luteus and S. aureus, the test was performed at the level of $p<0.05$.

\section{Results and discussion}

ZnO-CTS-based NPs synthesis and characterization

\section{UV-Vis spectra}

The UV-Vis spectra obtained for different ZnO-CTS NPs prepared through nano spray drying and precipitation method are given in Fig. 1a, b. The results indicated the
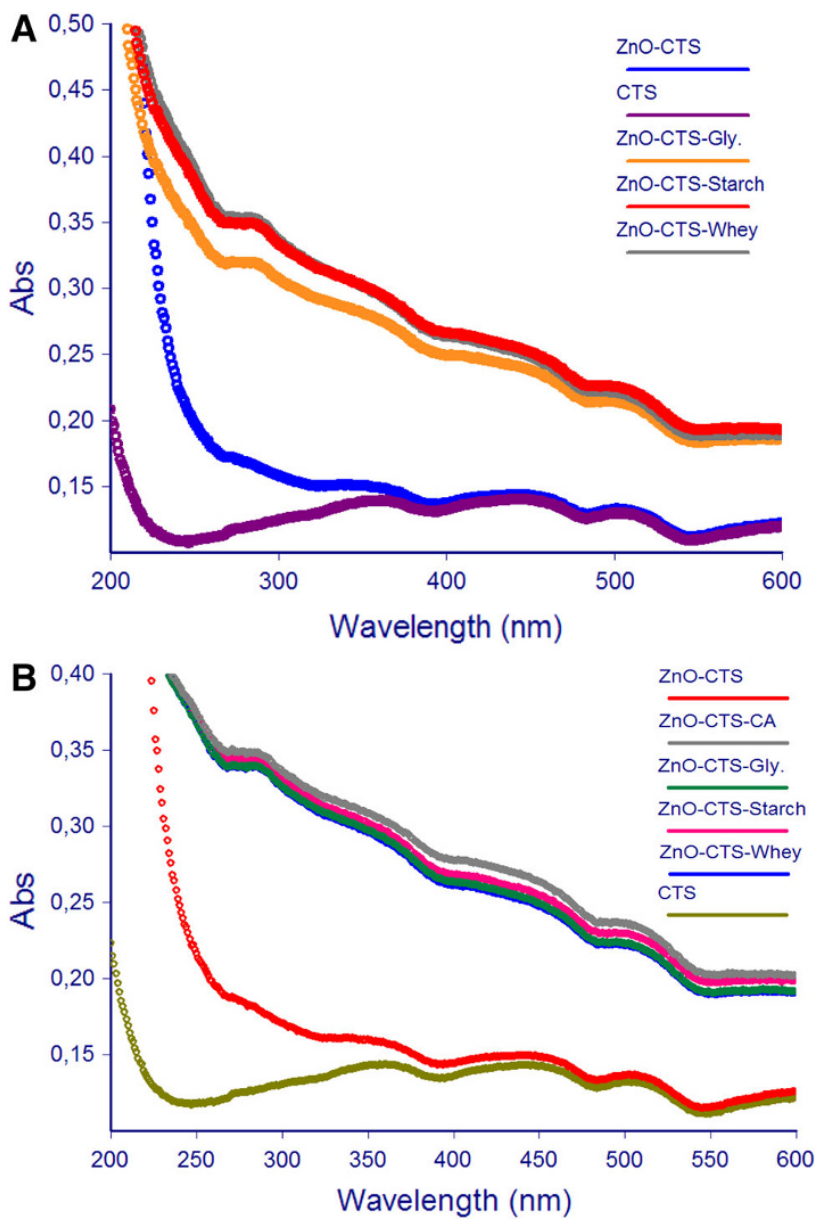

Fig. 1 UV-Vis spectra of the different ZnO-CTS NPs synthesized by: (a) nano spray drying and; (b) precipitation method

formation of ZnO-CTS NPs. The synthesis of ZnO-CTS NPs is clearly evident, as the excitation peak was observed due to ZnO-CTS NPs at $273 \mathrm{~nm}$, which lies much below the band-gap wavelength of $388 \mathrm{~nm}\left(E_{g}=3.2 \mathrm{eV}\right)$ of $\mathrm{ZnO}$. It was observed that absorption peaks of $\mathrm{ZnO} \mathrm{NPs}$ prepared in the presence of stabilizers were prominent as compared to ZnO-CTS NPs without stabilizers. The absorption peak for a suspension of NPs is broader and determined by the distribution of particle size. In the smaller wavelength range, particles with smaller size contribute more and at the region of absorbance maximum, all particles contribute to the absorbance [26]. Therefore, the average particle size of ZnO-CTS NPs observed in the presence of stabilizers was lower as compared to the $\mathrm{ZnO}$ NPs prepared in the absence of stabilizers.

\section{Size and zeta potential}

The mean size and size distribution of various $\mathrm{ZnO}-\mathrm{CTS}$ NPs with different stabilizers and synthesized through different methods are provided in Table 1 . The mean size 
Table 1 The mean size and size distribution measurements of the different $\mathrm{ZnO}-\mathrm{CTS}$ NPs

CA Citric acid, CTS chitosan, $P D I$ poly dispersity index

\begin{tabular}{lcccccccc}
\hline Sample & $\begin{array}{l}\text { Z-average } \\
(\mathrm{nm})\end{array}$ & PDI & Peak 1 & $\begin{array}{l}\text { Intensity } \\
(\%)\end{array}$ & Peak 2 & $\begin{array}{l}\text { Intensity } \\
(\%)\end{array}$ & Peak 3 & $\begin{array}{l}\text { Intensity } \\
(\%)\end{array}$ \\
\hline Nano spray drying method & & & & & & & & \\
ZnO (nano) Ctrl & 215.4 & 0.54 & 186.4 & 76.2 & 228.3 & 23.0 & - & - \\
ZnO + CTS & 167.2 & 0.64 & 148.9 & 92.5 & 268.4 & 7.50 & - & - \\
ZnO + CTS + glycerol & 402.5 & 0.89 & 1196 & 85.0 & 9.29 & 14.4 & - & - \\
ZnO + CTS + starch & 93.2 & 0.37 & 121.1 & 93.3 & 215.5 & 6.7 & - & - \\
ZnO +CTS + whey powder & 98.4 & 0.30 & 139.2 & 100 & - & - & & \\
Precipitation method & & & & & & & & \\
ZnO (nano) Ctrl & 603.1 & 0.89 & 156.9 & 83.8 & 32.3 & 16.2 & - & - \\
ZnO + CTS & 358.7 & 0.57 & 278.4 & 69.7 & 43.2 & 24.6 & 5.65 & 5.7 \\
ZnO +CTS + CA & 99.3 & 0.64 & 110.3 & 52.8 & 62.2 & 46.5 & 2.27 & 0.6 \\
ZnO + CTS + glycerol & 162.7 & 0.42 & 217.2 & 72.1 & 29.5 & 17.2 & 8.34 & 9.4 \\
ZnO + CTS + starch & 285.1 & 0.54 & 344.2 & 65.4 & 55.5 & 17.0 & 13.9 & 10.5 \\
ZnO +CTS + whey powder & 166.5 & 0.54 & 285.5 & 93.1 & 104.1 & 6.9 & - & - \\
\hline
\end{tabular}

of the ZnO-CTS NPs synthesized through nano spray dying method ranged between 93.2 and $403 \mathrm{~nm}$. The mean size of ZnO-CTS-glycerol NPs $(402.5 \mathrm{~nm})$ was even larger than the control ( $\mathrm{ZnO}-\mathrm{NPs}, 215 \mathrm{~nm}$ ). The larger size of NPs may be due to the aggregation of NPs which affects their mean size. The concrete reason for aggregation is not clearly understood. The size distribution is also highly variable with peak $1(1,196 \mathrm{~nm}$ with intensity of nearly $85 \%$ ) and peak $2(9.29 \mathrm{~nm}$ with intensity of $14.4 \%)$. This indicates that the concentration of $\mathrm{ZnO}$, CTS and glycerol needs to be optimized to get discrete, uniform and dispersed NPs. In all other treatments, there is not much variation between the mean size and size distribution.

The mean size of ZnO-CTS NPs with different stabilizers and synthesized through precipitation lies between 99.3 and $603 \mathrm{~nm}$. The $\mathrm{ZnO}-\mathrm{CTS}$ followed by $\mathrm{ZnO}-\mathrm{CTS}-$ starch exhibits the large size NPs with diameter of 359 and $285 \mathrm{~nm}$, respectively. The ZnO-CTS NPs formulation showed some cross linking or aggregation which impacts their mean size and size distribution. There was not much variation between the mean size and size distribution in other treatments. The NPs obtained in the present study can be further tailored with specific size, uniformity and highly dispersed nature befitted for specific applications through optimization of different variables, such as concentration of metal, CTS and stabilizers.

The zeta potential values of $-7.54,-12.9,-1.90$, -24.7 and $-35.5 \mathrm{mV}$ were calculated for $\mathrm{ZnO}, \mathrm{ZnO}-\mathrm{CTS}$, ZnO-CTS-glycerol, ZnO-CTS-starch and ZnO-CTSwhey NPs synthesized through nano spray drying method, respectively. Similarly, NPs fabricated via precipitation method resulted in zeta potential values of $-17.9,-2.68$, $-37.3,1.5,-12.8$ and $-11.0 \mathrm{mV}$ for $\mathrm{ZnO}, \mathrm{ZnO}-\mathrm{CTS}$, ZnO-CTS-CA, ZnO-CTS-glycerol, ZnO-CTS-starch and ZnO-CTS-whey NPs, respectively. Higher zeta potential value observed for NPs was prepared in the presence of different stabilizer except for ZnO-CTS-glycerol NPs in both methods. Higher zeta potential values can be due to the higher intensity of smaller size NPs in the medium with different stabilizers. Generally, the particles come closer and flocculation (aggregation of particles to form large size particle) takes place at low zeta potential and vice versa. Based on DLVO (Derjaguin, Landau, Verwey, and Overbeek) theory, a higher (negative) value of zeta potential is expected to result in larger electrostatic repulsion within the particles, leading to a higher shear sensitivity between particles and, consequently, smaller particle sizes.

\section{SEM of NPs formed through nano spray drying and precipitation method}

The scanning electron micrographs for ZnO-CTS NPs prepared by nano spray drying and precipitation method using different stabilizers are given in Figs. 2a-f, 3a-f. As evident from the micrographs, the NPs with different size and shapes (circular, elliptical and nano rods) were synthesized depending on the different treatments and methods.

As evident from the results, $\mathrm{ZnO}$ NPs synthesized by the nano spray drying method were agglomerated and rod type. It can be inferred that in the absence of CTS and stabilizer, the resultant $\mathrm{ZnO}$ NPs do not appear as discrete nanoscale particles and form much bulk dendritic floc-like structures with varying density. This type of aggregation was mainly due to the high surface energy of $\mathrm{ZnO}$ NPs [21] leading to formation of bulk structures with lower reactivity. On the contrary, ZnO-CTS NPs were observed to be more distinct and uniform as compared to ZnO NPs. The ZnO-CTS-CA NPs were not synthesized using nano spray drying process. The reason can be the formation of viscous solution 

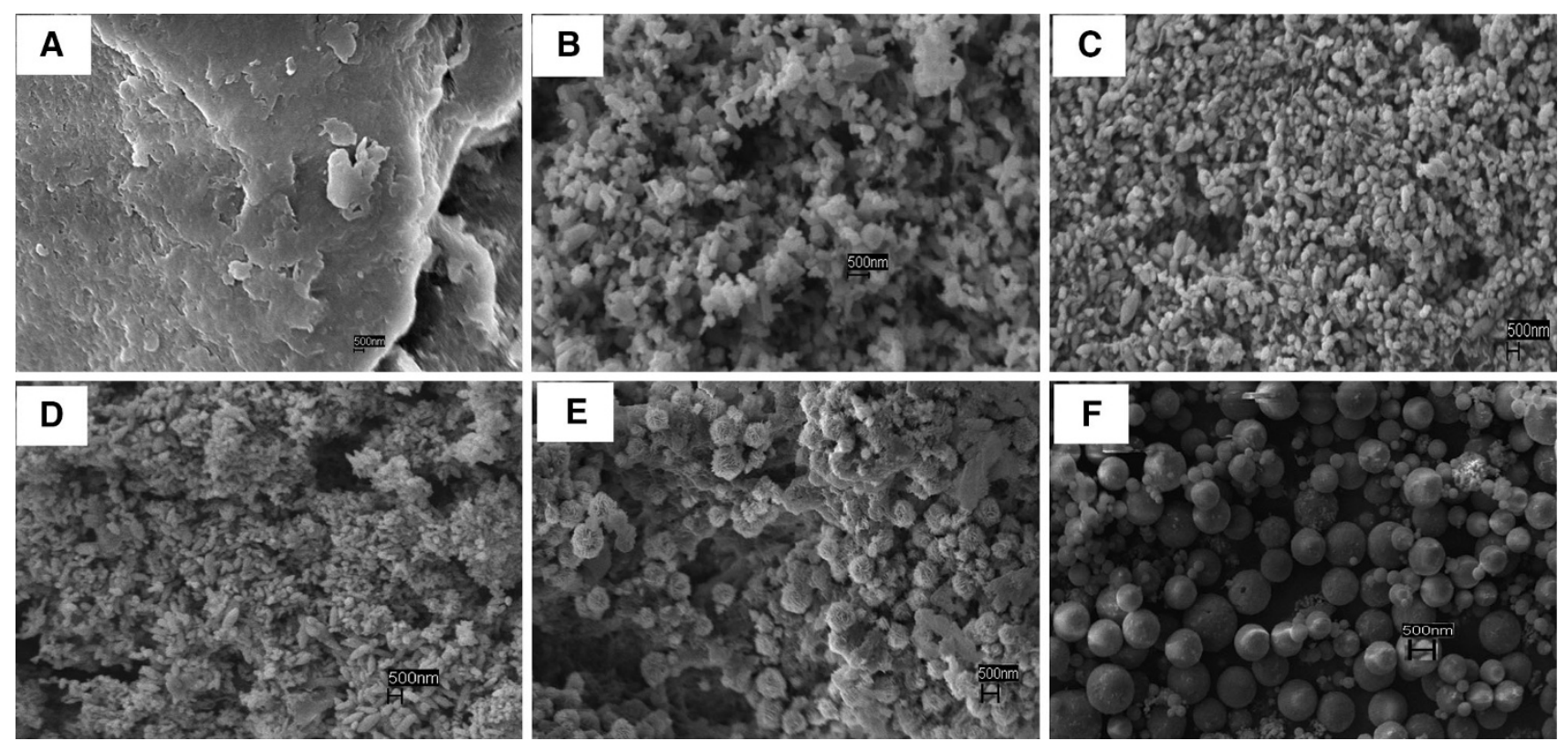

Fig. 2 SEM micrographs of: (a) chitosan (powder) and different NPs prepared through nano spray drying process: (b) ZnO-NPs; (c) ZnO-CTS; (d) ZnO-CTS-glycerol; (e) ZnO-CTS-starch and; (f) ZnO-CTS-whey powder. All the SEM images were taken at $10 \mathrm{~K}$ magnification
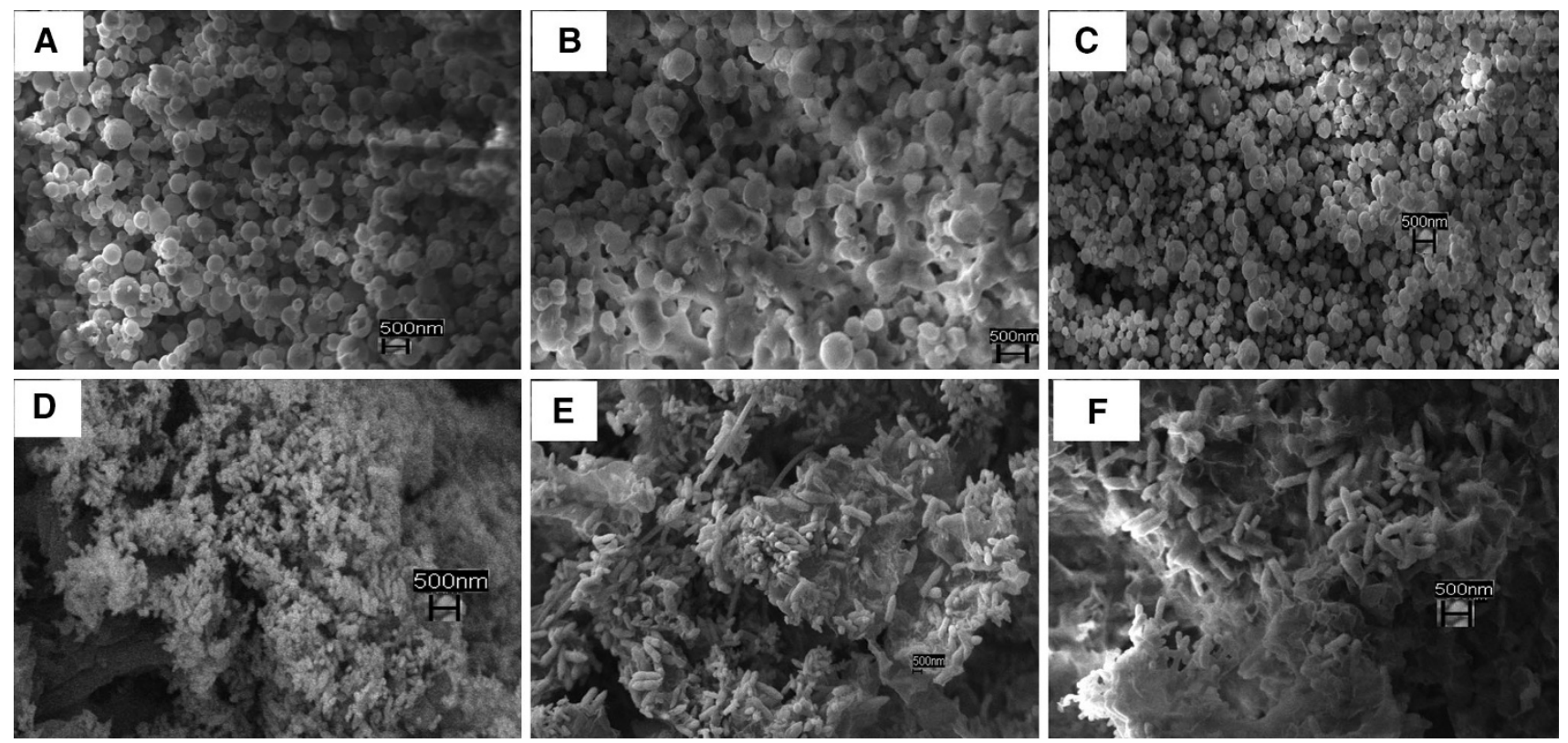

Fig. 3 SEM micrographs of different NPs prepared through chemical method: (a) ZnO-NPs; (b) ZnO-CTS; (c) ZnO-CTS-CA; (d) ZnO-CTSglycerol; (e) $\mathrm{ZnO}-\mathrm{CTS}$-starch and; (f) $\mathrm{ZnO}-\mathrm{CTS}-$ whey powder

(Viscosity $4.50 \mathrm{mPa} \mathrm{s}$ ) indicative of some polymer formation due to the presence of various functional groups on both CA and CTS resulting in some interaction between them. The nozzle of the nano spray dryer was blocked with viscous solution of $\mathrm{ZnO}-\mathrm{CTS}-\mathrm{CA}$ which resulted in the formation of thin film on the nozzle exit. ZnO-CTSglycerol NPs were also found to be less discrete and elliptical type. $\mathrm{ZnO}-\mathrm{CTS}-$ starch and $\mathrm{ZnO}-\mathrm{CTS}-$ whey stabilized NPs were observed to be circular in shape. $\mathrm{ZnO}-$ CTS-starch NPs were porous structures whereas $\mathrm{ZnO}-$ CTS-whey NPs were found to be more discrete and well dispersed as compared to ZnO-CTS-starch NPs. The presence of whey contributed to the steric hindrance between $\mathrm{ZnO}-\mathrm{CTS}$ NPs and prevented their aggregation and thus maintained the high surface area and reactivity of the particle. 
In comparison, $\mathrm{ZnO}$ NPs formed by precipitation technique (Fig. 3a-f) were discrete and less variable as compared to $\mathrm{ZnO}$ NPs formed through nano spray drying method. ZnO-CTS NPs formed by precipitation were circular but agglomerated. ZnO-CTS-CA NPs were found to be uniform, distinct and very well dispersed. ZnO-CTSglycerol NPs appeared to be bulk dendritic floc-like structures with non-discrete appearance. On the other hand, $\mathrm{ZnO}-\mathrm{CTS}-$ starch and $\mathrm{ZnO}-\mathrm{CTS}-$ whey NPs were found to possess rod-like aggregated structures. Studies demonstrated the multifunctional role of citrate ions in the NPs synthesis process, such as acting as a reducing agent, a stabilizer, and a complex agent [27, 28]. Ji et al. [27] suggested that the citrate ions act as reducing agents, stabilizers, and $\mathrm{pH}$ mediators in the synthesis of gold NPs. Various studies have shown that the growth of silver nanocrystals in solution is sensitive to the presence of CA or sodium citrate. However, the precise role of citrate ions still remains dubious. For instance, studies demonstrated that the citrate ions act as stabilizers in an efficient synthesis method for generating mono-dispersed silver nanoprisms through illumination of visible light $[29,30]$.

\section{Applications of ZnO-CTS NPs}

Antimicrobial activity

\section{Qualitative antimicrobial assay}

The results of qualitative antimicrobial assay using $\mathrm{ZnO}$ CTS NPs are provided in Table 2. As evident from the inhibition zone diameter measurements, it can be inferred that the ZnO-CTS NPs were more effective towards $M$. luteus and S. aureus as compared to fungal strain C. albicans. Based on the qualitative antimicrobial assay results, $M$. luteus and $S$. aureus were further selected for quantitative assay and biofilm inhibiting activity testing.

Quantitative antimicrobial assay

The antimicrobial activity of ZnO-CTS NPs against $M$. luteus and $S$. aureus is shown in Tables 3 and 4 . As evident from decrease in the OD $(600 \mathrm{~nm})$, the significant $(p<0.05)$ inhibition of growth was observed for M. luteus and S. aureus using $\mathrm{ZnO}-\mathrm{CTS}$ NPs prepared through both nano spray drying and precipitation method. ZnO-CTS NPS showed maximum inhibition at lower concentrations $(0.625-0.156 \mathrm{mg} / \mathrm{ml})$ $(p<0.05)$ against both M. luteus and S. aureus. However, increasing the concentration of $\mathrm{ZnO}-\mathrm{CTS}$ NPS above $0.625 \mathrm{mg} / \mathrm{ml}$, lower growth inhibition was observed.

ZnO-CTS NPs prepared by nano spray drying method showed 51.6-73.0 \% growth inhibition of M. luteus at
Table 2 Qualitative antimicrobial assay using agar diffusion method

\begin{tabular}{|c|c|c|c|}
\hline \multirow{2}{*}{$\begin{array}{l}\text { Tested samples } \\
(5 \mathrm{mg} / \mathrm{ml})\end{array}$} & \multicolumn{3}{|c|}{ Inhibition zone diameter (mm) } \\
\hline & $\begin{array}{l}\text { Candida } \\
\text { albicans }\end{array}$ & $\begin{array}{l}\text { Micrococcus } \\
\text { luteus }\end{array}$ & $\begin{array}{l}\text { Staphylococcus } \\
\text { aureus }\end{array}$ \\
\hline \multicolumn{4}{|l|}{ Control } \\
\hline $\mathrm{ZnO}$ powder & $<0.10$ & $<0.10$ & $<0.10$ \\
\hline CTS & 0.10 & 0.15 & 0.10 \\
\hline \multicolumn{4}{|c|}{ NPs synthesized by nano spray drying technique } \\
\hline $\mathrm{ZnO}-\mathrm{NPs}$ & 0.15 & 0.20 & 0.20 \\
\hline $\mathrm{ZnO}-\mathrm{CTS}$ NPs & 0.2 & 0.20 & 0.25 \\
\hline ZnO-CTS-glycerol & 0.2 & 0.30 & 0.30 \\
\hline $\mathrm{ZnO}-\mathrm{CTS}-$ starch & 0.2 & 0.25 & 0.35 \\
\hline $\begin{array}{l}\mathrm{ZnO}-\mathrm{CTS} \text {-whey } \\
\text { powder }\end{array}$ & 0.2 & 0.30 & 0.25 \\
\hline \multicolumn{4}{|c|}{ NPs synthesized by precipitation technique } \\
\hline $\mathrm{ZnO}-\mathrm{NPs}$ & 1.5 & 2.0 & 0.25 \\
\hline $\mathrm{ZnO}-\mathrm{CTS} \mathrm{NPs}$ & 2.0 & 2.5 & 0.30 \\
\hline $\mathrm{ZnO}-\mathrm{CTS}-\mathrm{CA}$ & 2.0 & 0.40 & 0.45 \\
\hline ZnO-CTS-glycerol & 1.5 & 0.25 & 0.25 \\
\hline ZnO-CTS-starch & $<2.0$ & 0.25 & 0.30 \\
\hline $\begin{array}{l}\text { ZnO-CTS-whey } \\
\text { powder }\end{array}$ & 2.0 & 0.25 & 0.25 \\
\hline
\end{tabular}

concentration of $0.156 \mathrm{mg} / \mathrm{ml}$. Similarly, ZnO-CTS NPs prepared by precipitation method showed 56.8-73.7\% growth inhibition of $M$. luteus at concentration of $0.156 \mathrm{mg} / \mathrm{ml}$. Significant $(p<0.05)$ growth inhibition of $S$. aureus was observed with $\mathrm{ZnO}-\mathrm{CTS}$ NPs concentration of $0.156 \mathrm{mg} / \mathrm{ml}$ as compared to higher concentrations. $\mathrm{ZnO}-$ CTS NPs prepared by nano spray drying and precipitation method showed growth inhibition of 62.0-73.4 and $65.2-82.0 \%$, respectively, for $S$. aureus at concentration of $0.156 \mathrm{mg} / \mathrm{ml}$. ZnO-CTS CA NPs prepared by precipitation method showed promising results with nearly $82 \%$ growth inhibition at concentration of $0.156 \mathrm{mg} / \mathrm{ml}$. No significant $(p>0.05)$ differences in M. luteus and $S$. aureus growth inhibition were observed between $\mathrm{ZnO}-\mathrm{CTS}$ NPs prepared by two different methods, namely nano spray drying and precipitation method.

Unlike ZnO-CTS NPs, CTS and $\mathrm{ZnO}$ powder exerted their growth inhibition effect at higher concentration of $5.0 \mathrm{mg} / \mathrm{ml}$. Significant $(p<0.05)$ inhibition of growth was observed for both $M$. luteus and S. aureus at higher dose of $5.0 \mathrm{mg} / \mathrm{ml}$ for CTS and $\mathrm{ZnO}$ powder. However, decreasing the concentration of CTS and $\mathrm{ZnO}$ powder to $0.625 \mathrm{mg} / \mathrm{ml}$ and below showed non-significant $(p>0.05)$ inhibition. The growth inhibition showed by bulk $\mathrm{ZnO}$ and CTS powder at higher concentrations of $5 \mathrm{mg} / \mathrm{ml}$ was found to be significantly lower than $\mathrm{ZnO}-\mathrm{CTS}$ NPs.

An important aspect of the use of $\mathrm{ZnO}$ as an antibacterial agent is its non-toxic nature towards human cells 


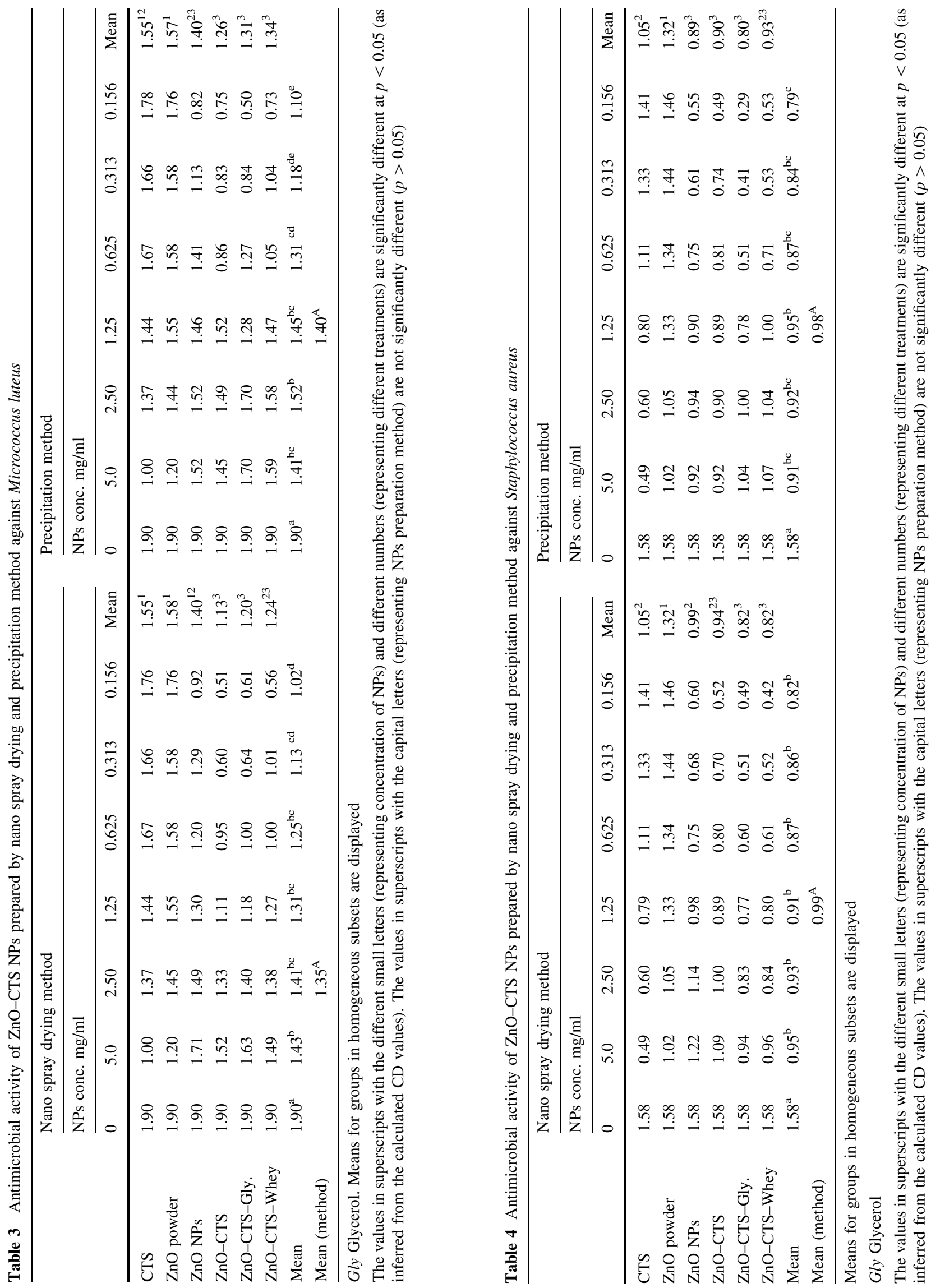


Table 5 Antibiofilm activity of ZnO-CTS NPs prepared by nano spray drying and precipitation method against Micrococcus luteus

\begin{tabular}{|c|c|c|c|c|c|c|c|c|c|c|c|c|c|c|c|c|}
\hline & \multicolumn{8}{|c|}{ Nano spray drying method } & \multicolumn{8}{|c|}{ Precipitation method } \\
\hline & \multicolumn{8}{|c|}{ NPs conc. $\mathrm{mg} / \mathrm{ml}$} & \multicolumn{8}{|c|}{ NPs conc. $\mathrm{mg} / \mathrm{ml}$} \\
\hline & 0 & 5.0 & 2.50 & 1.25 & 0.625 & 0.313 & 0.156 & Mean & 0 & 5.0 & 2.50 & 1.25 & 0.625 & 0.313 & 0.156 & Mean \\
\hline CTS & 1.43 & 0.76 & 0.86 & 0.96 & 0.98 & 1.07 & 1.28 & $1.05^{2}$ & 1.43 & 0.76 & 0.86 & 0.96 & 0.98 & 1.07 & 1.08 & $1.02^{2}$ \\
\hline $\mathrm{ZnO}$ powder & 1.43 & 0.93 & 0.91 & 1.01 & 1.25 & 1.36 & 1.39 & $1.18^{1}$ & 1.43 & 0.93 & 0.91 & 1.01 & 1.25 & 1.36 & 1.39 & $1.18^{1}$ \\
\hline ZnO NPs & 1.43 & 0.82 & 0.80 & 0.69 & 0.60 & 0.58 & 0.58 & $0.79^{3}$ & 1.43 & 0.93 & 0.90 & 0.78 & 0.70 & 0.58 & 0.48 & $0.83^{3}$ \\
\hline $\mathrm{ZnO}-\mathrm{CTS}$ & 1.43 & 0.69 & 0.65 & 0.64 & 0.62 & 0.54 & 0.54 & $0.73^{3}$ & 1.43 & 0.75 & 0.73 & 0.70 & 0.65 & 0.54 & 0.45 & $0.75^{34}$ \\
\hline ZnO-CTS-Gly & 1.43 & 0.62 & 0.59 & 0.58 & 0.48 & 0.48 & 0.48 & $0.67^{3}$ & 1.43 & 0.60 & 0.58 & 0.55 & 0.53 & 0.44 & 0.33 & $0.64^{4}$ \\
\hline $\begin{array}{l}\text { ZnO-CTS- } \\
\text { Whey }\end{array}$ & 1.43 & 0.75 & 0.65 & 0.58 & 0.58 & 0.57 & 0.55 & $0.73^{3}$ & 1.43 & 0.65 & 0.63 & 0.59 & 0.57 & 0.50 & 0.41 & $0.68^{4}$ \\
\hline Mean & $1.43^{\mathrm{a}}$ & $0.76^{\mathrm{b}}$ & $0.74^{\mathrm{b}}$ & $0.74^{\mathrm{b}}$ & $0.75^{\mathrm{b}}$ & $0.77^{\mathrm{b}}$ & $0.80^{\mathrm{b}}$ & & $1.43^{\mathrm{a}}$ & $0.77^{\mathrm{b}}$ & $0.77^{\mathrm{b}}$ & $0.77^{\mathrm{b}}$ & $0.78^{\mathrm{b}}$ & $0.75^{\mathrm{b}}$ & $0.69^{\mathrm{b}}$ & \\
\hline Mean (method) & & & $0.86^{\mathrm{A}}$ & & & & & & & & & $0.85^{\mathrm{A}}$ & & & & \\
\hline
\end{tabular}

Means for groups in homogeneous subsets are displayed

Gly Glycerol

The values in superscripts with the different small letters (representing concentration of NPs) and different numbers (representing different treatments) are significantly different at $p<0.05$ (as inferred from the calculated CD values). The values in superscripts with the capital letters (representing NPs preparation method) are not significantly different $(p>0.05)$

which is also the prerequisite for any antimicrobial agent [31]. $\mathrm{ZnO}$ is currently being investigated as an antibacterial agent in both micro-scale and nanoscale formulations. Various studies have demonstrated that $\mathrm{ZnO}$ NPs showed antibacterial activity [32-39]. As evident from this study, the antimicrobial activities displayed by $\mathrm{ZnO}$ NPs were higher as compared to micro particles [39]. The exact mechanisms of the antibacterial action have not yet been clearly elucidated. However, research advocated various mechanisms, such as the role of reactive oxygen species (ROS) generated on the surface of the particles [40], zinc ion release [36], membrane dysfunction [36, 37], and NPs internalization [33]. In the present study, the growth inhibition of both M. luteus and S. aureus with ZnO-CTS NPs can be attributed to the above mentioned mechanisms exerted by the $\mathrm{ZnO}$ NPs.

\section{Antibiofilm activity}

The biofilm inhibition activity of ZnO-CTS NPs against $M$. luteus and S. aureus is shown in Tables 5 and 6. ZnO-CTS NPs prepared through nano spray dying and precipitation methods showed significant $(p<0.05)$ biofilm inhibition activity against $M$. luteus and $S$. aureus at a lower concentration ranging from 0.625 to $0.156 \mathrm{mg} / \mathrm{ml}$.

For M. luteus at NPs concentration of $0.156 \mathrm{mg} / \mathrm{ml}$, ZnO-CTS NPs prepared by nano spray drying and precipitation method showed biofilm inhibition of 59.4-66.4 and 66.4-77.0\%, respectively. No significant difference $(p>0.05)$ was observed between different $\mathrm{ZnO}-\mathrm{CTS}$ NPs prepared by nano spray drying method for biofilm inhibition at NPs concentration ranging from 0.625 to $0.156 \mathrm{mg} /$ ml. However, significant difference was observed between $\mathrm{ZnO}$ NPs and ZnO-CTS NPs prepared by precipitation method on biofilm inhibition for M. luteus. Likewise, for $S$. aureus at NPs concentration of $0.156 \mathrm{mg} / \mathrm{ml}, \mathrm{ZnO}-\mathrm{CTS}$ NPs prepared by nano spray drying and precipitation method showed biofilm inhibition of 69.0-84.1 and $67.5-80.2 \%$ respectively. No significant difference $(p>0.05)$ was observed on biofilm inhibition between different $\mathrm{ZnO}-\mathrm{CTS}$ NPs prepared by nano spray drying and precipitation method at all NPs concentrations used. $\mathrm{ZnO}$ CTS-glycerol NPs and ZnO-CTS-CA NPs prepared by nano spray drying and precipitation method, respectively, showed maximum biofilm inhibition at concentration of $0.156 \mathrm{mg} / \mathrm{ml}$. No significant differences $(p>0.05)$ with respect to $M$. luteus and $S$. aureus biofilms inhibition were observed with NPs prepared by two different methods.

ZnO-CTS NPs showed higher biofilm inhibition activity against both bacterial strains as compared to micro particles of $\mathrm{ZnO}$ and CTS. Higher biofilm inhibition activity was achieved with lower concentration of NPs (ranging from 0.625 to $0.156 \mathrm{mg} / \mathrm{ml}$ ) as compared to micro particles ( $\mathrm{ZnO}$ and CTS powder) which exerted higher biofilm inhibition activity at higher concentrations of $5 \mathrm{mg} / \mathrm{ml}$.

Micrococcus luteus and Staphylococcus aureus represent two of the most common opportunistic pathogens, due to their frequent incidence in the etiology of the community-acquired and nosocomial infections and to their high rates of natural and acquired resistance to different antimicrobial agents including the widely used antibiotics. The clinical incidence of these bacterial strains is augmented due to their ability to colonize various industrial settings 
Table 6 Antibiofilm activity of ZnO-CTS NPs prepared by nano spray drying and precipitation method against Staphylococcus aureus

\begin{tabular}{|c|c|c|c|c|c|c|c|c|c|c|c|c|c|c|c|c|}
\hline & \multicolumn{8}{|c|}{ Nano spray drying method } & \multicolumn{8}{|c|}{ Precipitation method } \\
\hline & \multicolumn{8}{|c|}{ NPs conc. mg/ml } & \multicolumn{8}{|c|}{ NPs conc. $\mathrm{mg} / \mathrm{ml}$} \\
\hline & 0 & 5.0 & 2.50 & 1.25 & 0.625 & 0.313 & 0.156 & Mean & 0 & 5.0 & 2.50 & 1.25 & 0.625 & 0.313 & 0.156 & Mean \\
\hline CTS & 1.26 & 0.67 & 0.76 & 0.79 & 0.82 & 0.94 & 0.98 & $0.89^{1}$ & 1.26 & 0.67 & 0.76 & 0.79 & 0.82 & 0.94 & 0.98 & $0.89^{1}$ \\
\hline $\mathrm{ZnO}$ powder & 1.26 & 0.73 & 0.80 & 0.84 & 0.90 & 0.97 & 1.05 & $0.94^{1}$ & 1.26 & 0.73 & 0.79 & 0.84 & 0.89 & 0.97 & 1.05 & $0.93^{1}$ \\
\hline $\mathrm{ZnO}$ NPs & 1.26 & 0.64 & 0.61 & 0.60 & 0.55 & 0.45 & 0.39 & $0.64^{2}$ & 1.26 & 0.62 & 0.64 & 0.59 & 0.45 & 0.42 & 0.41 & $0.63^{2}$ \\
\hline $\mathrm{ZnO}-\mathrm{CTS}$ & 1.26 & 0.59 & 0.54 & 0.48 & 0.42 & 0.38 & 0.30 & $0.57^{2}$ & 1.26 & 0.55 & 0.49 & 0.43 & 0.42 & 0.41 & 0.33 & $0.56^{2}$ \\
\hline ZnO-CTS-Gly. & 1.26 & 0.65 & 0.58 & 0.44 & 0.40 & 0.32 & 0.20 & $0.55^{2}$ & 1.26 & 0.54 & 0.55 & 0.53 & 0.45 & 0.31 & 0.25 & $0.56^{2}$ \\
\hline $\begin{array}{l}\text { ZnO-CTS- } \\
\text { Whey }\end{array}$ & 1.26 & 0.69 & 0.64 & 0.55 & 0.46 & 0.36 & 0.35 & $0.62^{2}$ & 1.26 & 0.57 & 0.49 & 0.47 & 0.46 & 0.35 & 0.31 & $0.56^{2}$ \\
\hline Mean & $1.26^{\mathrm{a}}$ & $0.66^{\mathrm{b}}$ & $0.65^{\mathrm{b}}$ & $0.62^{\mathrm{b}}$ & $0.59^{\mathrm{b}}$ & $0.57^{\mathrm{b}}$ & $0.54^{\mathrm{b}}$ & & $1.26^{\mathrm{a}}$ & $0.61^{\mathrm{b}}$ & $0.62^{\mathrm{b}}$ & $0.61^{\mathrm{b}}$ & $0.58^{\mathrm{b}}$ & $0.57^{\mathrm{b}}$ & $0.55^{\mathrm{b}}$ & \\
\hline Mean (method) & & & & $0.70^{\mathrm{A}}$ & & & & & & & & $0.69^{\mathrm{A}}$ & & & & \\
\hline
\end{tabular}

Means for groups in homogeneous subsets are displayed

Gly Glycerol

The values in superscripts with the different small letters (representing concentration of NPs) and different numbers (representing different treatments) are significantly different at $p<0.05$ (as inferred from the calculated CD values). The values in superscripts with the capital letters (representing NPs preparation method) are not significantly different $(p>0.05)$

and the cellular as well as the inert substrates, such as medical devices. Both these strains are responsible for the formation of biofilms on medical devices and are associated with various chronic infections which are very hard to treat and often are responsible for severe outcome [41]. In the present study, the inhibitory effect on biofilm could be explained by the potential inhibitory effect of the ZnO NPs on the bacterial exopolysaccharides secretion. Moreover, both $\mathrm{ZnO}$ and CTS showed antimicrobial properties. These results are accounting for the potential use of $\mathrm{ZnO}-\mathrm{CTS}$ NPs in the prevention of the bacterial biofilm development on prosthetic devices, as well as for the design of new antiseptics and disinfectants with efficient protective action against bacterial colonization of tissue and inert surfaces. Further, different types of formulations can be developed for these NPs through encapsulation, powder and hydrogels to suit different application needs.

\section{Conclusions}

The study demonstrated the facile synthesis of $\mathrm{ZnO}-\mathrm{CTS}$ nanoparticles stabilized with different organic compounds. The synthesis of NPs was carried out by two different methods: (1) nano spray drying and; (2) precipitation method. The size distribution analysis, UV-Vis spectrum and SEM monographs of ZnO-CTS NPs indicated that NPs prepared in the presence of stabilizers agglomerated less and dispersed better in water as compared to $\mathrm{ZnO}$ and ZnO-CTS NPs without stabilizers. Both the synthesis methods were simple and were devoid of any chemical usage. However, the ratios of $\mathrm{ZnO}, \mathrm{CTS}$ and stabilizer need to be further optimized for uniform size, shape and better dispersed NPs. The ZnO-CTS NPs showed significant antimicrobial activity and biofilm inhibition activity against $M$. luteus and $S$. aureus. The study indicated the promising potential of $\mathrm{ZnO}-\mathrm{CTS}$ NPs as antimicrobial and biofilm inhibition agents.

Acknowledgments The authors are sincerely thankful to the Natural Sciences and Engineering Research Council of Canada (Discovery Grant 355254) and Initiative Inde 2010 (MELS) for financial support. The views or opinions expressed in this article are those of the authors.

Conflict of interest The authors declare that they have no competing interests.

Open Access This article is distributed under the terms of the Creative Commons Attribution License which permits any use, distribution, and reproduction in any medium, provided the original author(s) and the source are credited.

\section{References}

1. Wei, A., Sun, X.W., Wang, J.X., Lei, Y., Cai, X.P., Li, C.M., Dong, Z.L., Huang, W.: Enzymatic glucose biosensor based on $\mathrm{ZnO}$ nano rods grown by hydrothermal decomposition. Appl. Phys. Lett. 89(12), 123902 (2006)

2. Wang, J.X., Sun, X.W., Wei, A., Lei, Y., Cai, X.P., Li, C.M., Dong, Z.L.: Zinc oxide nano comb biosensor for glucose detection. Appl. Phys. Lett. 88(23), 233106-233108 (2006)

3. Singh, S.P., Arya, S.K., Pandey, P., Malhotra, B.D., Saha, S., Sreenivas, K., Gupta, V.: Cholesterol biosensor based on sputtered zinc oxide nano porous thin film. Appl. Phys. Lett. 91(1-3), 063901 (2007) 
4. Cheng, J.P., Zhang, X.B., Tao, X.Y., Lu, H.M., Luo, Z.Q., Liu, F.: Fine-tuning the synthesis of $\mathrm{ZnO}$ nanostructures by an alcohol thermal process. J. Phys. Chem. B 110(21), 10348-10353 (2006)

5. Wahab, R., Ansari, S.G., Kim, Y.S., Song, M., Shin, H.S.: The role of $\mathrm{pH}$ variation on the growth of zinc oxide nanostructures. Appl. Surf. Sci. 255(9), 4891-4896 (2009)

6. Zhang, J., Sun, L., Yin, J., Su, H., Liao, C., Yan, C.: Control of $\mathrm{ZnO}$ morphology via a simple solution route. Chem. Mater. 14(10), 4172-4177 (2002)

7. Ayudhya, S.K.N., Tonto, P., Mekasuwandumrong, O., Pavarajarn, V., Praserthdam, P.: Solvothermal synthesis of $\mathrm{ZnO}$ with various aspect ratios using organic solvents. Cryst. Growth Des. 6(11), 2446-2450 (2006)

8. Bitenc, M., Podbrscek, P., Orel, Z.C., Cleveland, M.A., Paramo, J.A., Peters, R.M., Strzhemechny, Y.M.: Correlation between morphology and defect luminescence in precipitated $\mathrm{ZnO}$ nanorod powders. Cryst. Growth Des. 9(2), 997-1001 (2009)

9. Ozgur, U., Alivov, Y.I., Liu, C., Teke, A., Reshchikov, M.A., Dogan, S., Avrutin, V., Cho, S.J., Morkoc, H.: A comprehensive review of $\mathrm{ZnO}$ materials and devices. J. Appl. Phys. 98(4), 041301-041303 (2005)

10. Kim, J.Y., Osterloh, F.E.: ZnO-CdSe nanoparticles clusters as directional photo emitters with tunable wavelength. J. Am. Chem. Soc. 127(29), 10152-10153 (2005)

11. Hubbard, N.B., Culpepper, M.L., Howell, L.L.: Actuators for micro positioners and nano positioners. Appl. Mech. Rev. 59(1-6), 324-334 (2006)

12. Lee, H.J., Yeo, S.Y., Jeong, S.H.: Antibacterial effect of nano sized silver colloidal solution on textile fabrics. J. Mater. Sci. 38(10), 2199-2204 (2003)

13. Wang, L., Muhammed, M.: Synthesis of zinc oxide nanoparticles with controlled morphology. J. Mater. Chem. 9(11), 2871-2878 (1999)

14. Xu, H.Y., Wang, H., Zhang, Y.C.: Hydrothermal synthesis of zinc oxide powders with controllable morphology. Ceramics Inter 30(1), 93-97 (2004)

15. Tani, T., Mdler, L., Pratsinis, S.E.: Homogeneous ZnO nanoparticles by flame spray pyrolysis. J Nanoparticle Res 4(4), 337-343 (2002)

16. Li, L.H., Deng, J.C., Deng, H.R., Liu, Z.L., Xin, L.: Synthesis and characterization of chitosan $\mathrm{ZnO}$ nanoparticle composite membranes. Carbohyd Res 345(8), 994-998 (2010)

17. Agnihotri, S.A., Mallikarjuna, N.N., Aminabhavi, T.M.: Recent advances on chitosan-based micro- and nanoparticles in drug delivery. J. Controlled Release 100(1), 5-28 (2004)

18. Kim, S.K., Rajapakse, N.: Enzymatic production and biological activities of chitosan oligosaccharides (COS): a review. Carbohyd Polym 62(4), 357-368 (2005)

19. Muzzarelli, R.A.A., Sipos, L.: Chitosan for the collection from seawater of naturally occurring zinc, cadmium, lead and copper. Talanta 18(9), 853-858 (1971)

20. Posthumus, W., Magusin, P.C.M., Zijp, J.C.M.B., Tinnemans, A.H.A., Linde, R.: Surface modification of oxidic nanoparticles using 3-methacryloxypropyl-trimethoxysilane. J. Coll. Interf. Sci. 269, 109-116 (2004)

21. Tang, E., Cheng, G., Ma, X., Pang, X., Zhao, Q.: Surface modification of $\mathrm{ZnO}$ nanoparticles by PMAA and its dispersion in aqueous system. Appl. Surf. Sci. 252(14), 5227-5232 (2006)

22. Ryan, K.J., Ray, C.G. (ed.): Sherris Medical Microbiology (4th ed). McGraw Hill, New York, ISBN 0-8385-8529-9 (2004)

23. Bowersox, J.: Experimental staph vaccine broadly protective in animal studies. NIH. Archived from the original on 5 May 2007(1999)
24. Limban, C., Marutescu, L., Chifiriuc, M.C.: Synthesis, spectroscopic properties and antipathogenic activity of new thiourea derivatives. Molecules 16(9), 7593-7607 (2011)

25. Olar, R., Badea, M., Marinescu, D., Chifiriuc, M.C., Bleotu, C., Grecu, M.N., Iorgulescu, E.M., Bucur, M., Lazar, A., Finaru, A.: Prospects for new antimicrobials based on $N, N$ dimethyl biguanide complexes as effective agents on both planktonic and adhered microbial strains. Eur. J. Med. Chem. 45, 2868-2875 (2010)

26. Pesika, N.S., Stebe, K.J., Searson, P.C.: Determination of the particle size distribution of quantum nano crystals from absorbance spectra. Adv. Matter. 15(15), 1289-1296 (2003)

27. Ji, X., Song, X., Li, J., Bai, Y., Yang, W., Peng, X.: Size control of gold nano crystals in citrate reduction: the third role of citrate. J. Am. Chem. Soc. 129(45), 13939-13948 (2007)

28. Jiang, X.C., Chen, C.Y., Chen, W.M., Yu, A.B.: Role of citric acid in the formation of silver nano plates through a synergistic reduction approach. Langmuir 26(6), 4400-4408 (2010)

29. Jin, R., Cao, Y., Metraux, G.S., Schatz, G.C., Mirkin, C.A.: Controlling anistropic nanoparticle growth through plasmon excitation. Nature 425, 487-490 (2003)

30. Jin, R., Cao, Y., Mirkin, C.A., Kelly, K.L., Schatz, G.C., Zheng, J.G.: Photo induced conversion of silver nanospheres to nanoprisms. Sci 294(5548), 1901-1903 (2001)

31. Nair, S., Sasidharan, A., Divya Rani, V.V., Menon, D., Nair, S., Manzoor, K., Raina, S.: Role of size scale of $\mathrm{ZnO}$ nanoparticles and microparticles on toxicity toward bacteria and osteoblast cancer cells. J. Mater. Sci. Mater. Med. 20(S1), 235-241 (2009)

32. Adams, L.K., Lyon, D.Y., Alvarez, P.J.J.: Comparative eco-toxicity of nanoscale $\mathrm{TiO}_{2}, \mathrm{SiO}_{2}$, and $\mathrm{ZnO}$ water suspensions. Water Res. 40(19), 3527-3532 (2006)

33. Brayner, R., Ferrari-Iliou, R., Brivois, N., Djediat, S., Benedetti, M.F., Fiévet, F.: Toxicological impact studies based on Escherichia coli bacteria in ultrafine $\mathrm{ZnO}$ nanoparticles colloidal medium. Nano Lett. 6(4), 866-870 (2006)

34. Colon, G., Ward, B.C., Webster, T.J.: Increased osteoblast and decreased staphylococcus epidermidis functions on nanophase $\mathrm{ZnO}$ and $\mathrm{TiO}_{2}$. J. Biomed. Mater. Res. A78, 595-604 (2006)

35. Jeng, H.A., Swanson, J.: Toxicity of metal oxide nanoparticles in mammalian cells. J. Environ. Sci. Health A Tox. Hazard. Subst. Environ. Eng. 41(12), 2699-2711 (2006)

36. Yang, $\mathrm{Z}$., Xie, C.: $\mathrm{Zn}^{2+}$ release from zinc and zinc oxide particles in simulated uterine solution. Coll. Surf. B Biointerf. 47(2), 140-145 (2006)

37. Zhang, L., Jiang, Y., Ding, Y., Povey, M., York, D.: Investigation into the antibacterial behavior of suspensions of $\mathrm{ZnO}$ nanoparticles (ZnO nano fluids). J. Nano. Part. Res. 9(3), 479-489 (2007)

38. Reddy, K.M., Feris, K., Bell, J., Wingett, D.G., Hanley, C., Punnoose, A.: Selective toxicity of zinc oxide nanoparticles to prokaryotic and eukaryotic systems. Appl. Phys. Lett. 90(213902), 2139021-2139023 (2007)

39. Yamamoto, O.: Influence of particle size on the antibacterial activity of zinc oxide. Int. J. Inorg. Mater. 3(7), 643-646 (2001)

40. Sawai, J., Shouji, S., Igarashi, H., Hashimoto, A., Kokugan, T., Shimizu, M., Kojima, H.: Hydrogen peroxide as an antibacterial factor in zinc oxide powder slurry. J. Ferment. Bioeng. 86(5), 521-522 (1998)

41. Donlan, R.M., Costerton, J.W.: Biofilms: survival mechanisms of clinically relevant microorganisms. Clin. Microbiol. Rev. 15, 167-193 (2002) 